\title{
Study of GSLT in Curvature-Matter Coupling Gravity
}

\author{
Muhammad Sharif ${ }^{1, \star}$ and Ayesha Ikram $^{1, \star \star}$ \\ ${ }^{1}$ Department of Mathematics, University of the Punjab, Quaid-e-Azam Campus, Lahore-54590, Pakistan.
}

\begin{abstract}
In this work, we study the first and generalized second laws of thermodynamics at the apparent horizon of homogeneous and isotropic universe model in the context of $f(\mathcal{G}, T)$ gravity ( $\mathcal{G}$ and $T$ represent the Gauss-Bonnet invariant and trace of the energymomentum tensor, respectively). We formulate the corresponding field equations as well as determine the radius, temperature and entropy to analyze these laws. An extra term associated with entropy production is appeared in the first law due to the non-equilibrium treatment of thermodynamics. It is found that the universal condition is obtained to preserve the generalized second law of thermodynamics.
\end{abstract}

\section{Introduction}

Thermodynamics has been a subject of great interest to explore the fascinating characteristics of matter variables in general relativity (GR) as well as in modified gravitational theories. For isotropic and homogeneous universe, Einstein field equations can be expressed in terms of first law of thermodynamics (FLT) [1]. Akbar and Cai [2] found that Friedmann equations evaluated at the apparent horizon can be rewritten in the form $d \mathcal{E}=\tau d S+W d V(\mathcal{E}, \tau, S, V$ and $W$ are the energy, temperature, entropy, volume inside the horizon and work density, respectively) in the background of GR, GaussBonnet (GB) gravity and the general Lovelock theory. It is found that an auxiliary entropy production term corresponding to the non-equilibrium treatment of thermodynamics is appeared in Clausius relation in modified theories of gravity while no such additional term is obtained in braneworld, GB and Lovelock gravitational theories [3]. Wu et al. [4] formulated the universal condition to check the validity of generalized second law of thermodynamics (GSLT) in the context of modified theories of gravity. Sadjadi [5] explored the validity of second law and GSLT for power-law solution as well as de Sitter universe in $f(R, \mathcal{G})$ gravity. Abdolmaleki and Najafi [6] investigated GSLT in $f(\mathcal{G})$ gravity for isotropic and homogeneous universe filled with matter and radiation enclosed by apparent horizon with Hawking temperature.

In this paper, we explore the first and GSLT at the apparent horizon in $f(\mathcal{G}, T)$ gravity. This modified gravitational theory deals with the non-minimal coupling between quadratic curvature invariant (a linear combination of Ricci scalar $(R)$, Ricci $\left(R_{\alpha \beta}\right)$ and Riemann $\left(R_{\alpha \beta \xi \eta}\right)$ tensors) and matter. The paper has the following format. In the next section, we construct the corresponding field equations for isotropic and homogeneous universe with any spatial curvature while section $\mathbf{3}$ investigates the laws of thermodynamics at the apparent horizon of universe model. We summarize the results in the last section.

\footnotetext{
${ }^{\star}$ e-mail: msharif.math@pu.edu.pk

$\star \star$ e-mail: ayeshamaths91@gmail.com
} 


\section{$2 f(G, T)$ Gravity}

The action of $f(\mathcal{G}, T)$ gravity is given by [7]

$$
\mathcal{S}_{f(\mathcal{G}, T)}=\int d^{4} x \sqrt{-g}\left[\frac{1}{16 \pi G}(R+f(\mathcal{G}, T))+\mathcal{L}_{m}\right],
$$

where $\mathcal{G}=R_{\alpha \beta \xi \eta} R^{\alpha \beta \xi \eta}-4 R_{\alpha \beta} R^{\alpha \beta}+R^{2}, g, \mathcal{L}_{m}$ and $G$ denote determinant of the metric tensor $\left(g_{\alpha \beta}\right)$, Lagrangian density associated with matter configuration and gravitational constant, respectively. The suitable form of generic function $f(\mathcal{G}, T)$ describing the non-minimal coupling between curvature and matter is of the form

$$
f(\mathcal{G}, T)=f_{1}(\mathcal{G})+f_{2}(\mathcal{G}) f_{3}(T) .
$$

The variation of the action (1) with respect to $g_{\alpha \beta}$ for the above model in the presence of pressureless fluid yields the following field equations

$$
G_{\alpha \beta}=8 \pi \mathbb{G} T_{\alpha \beta}^{(\mathrm{eff})}=8 \pi \mathbb{G} T_{\alpha \beta}+T_{\alpha \beta}^{(\mathcal{G T})}
$$

where

$$
\begin{aligned}
T_{\alpha \beta}^{(\mathcal{G T})} & =\frac{1}{2} g_{\alpha \beta}\left(f_{1}(\mathcal{G})+f_{2}(\mathcal{G}) f_{3}(T)\right)-\left(2 R_{\alpha}^{\xi \eta \delta} R_{\beta \xi \eta \delta}-4 R_{\alpha \xi \beta \eta} R^{\xi \eta}-4 R_{\alpha}^{\xi} R_{\xi \beta}+2 R R_{\alpha \beta}\right) \\
& \times\left(f_{1}^{\prime}(\mathcal{G})+f_{2}^{\prime}(\mathcal{G}) f_{3}(T)\right)-\left(4 R_{\alpha \xi \beta \eta} \nabla^{\xi} \nabla^{\eta}-4 g_{\alpha \beta} R^{\xi \eta} \nabla_{\xi} \nabla_{\eta}+4 R_{\alpha}^{\xi} \nabla_{\beta} \nabla_{\xi}+4 R_{\beta}^{\xi} \nabla_{\alpha} \nabla_{\xi}\right. \\
& \left.-4 R_{\alpha \beta} \nabla^{2}-2 R \nabla_{\alpha} \nabla_{\beta}+2 R g_{\alpha \beta} \nabla^{2}\right)\left(f_{1}^{\prime}(\mathcal{G})+f_{2}^{\prime}(\mathcal{G}) f_{3}(T)\right), \\
\mathbb{G} & =G \mathfrak{B}, \quad \mathfrak{B}=1+\frac{f_{2}(\mathcal{G}) f_{3}^{\prime}(T)}{8 \pi G},
\end{aligned}
$$

$\nabla^{2}=\nabla_{\alpha} \nabla^{\alpha}\left(\nabla_{\alpha}\right.$ is a covariant derivative) and prime represents derivative with respect to the corresponding variable.

The line element for homogeneous and isotropic universe is

$$
d s^{2}=d t^{2}-\frac{a^{2}(t)}{1-\mathcal{K} r^{2}} d r^{2}-\chi^{2} d \theta^{2}-\chi^{2} \sin ^{2} \theta d \phi^{2},
$$

where $\chi=a(t) r, a(t)$ and $\mathcal{K}$ represent the scale factor and spatial curvature parameter associated with open, flat and closed cosmic geometries for $\mathcal{K}=-1,0$ and 1, respectively. Using Eqs.(3) and (4), we obtain

$$
\begin{aligned}
3\left(H^{2}+\frac{\mathcal{K}}{a^{2}}\right) & =8 \pi \mathbb{G} \rho^{(\mathrm{tot})}=8 \pi \mathbb{G}\left(\rho+\rho^{(\mathrm{hc})}\right), \\
-2\left(\dot{H}-\frac{\mathcal{K}}{a^{2}}\right) & =8 \pi \mathbb{G}\left(\rho^{(\mathrm{tot})}+p^{(\mathrm{tot})}\right)=8 \pi \mathbb{G}\left(\rho+\rho^{(\mathrm{hc})}+p^{(\mathrm{hc})}\right),
\end{aligned}
$$

where $\rho^{(\mathrm{hc})}$ and $p^{(\mathrm{hc})}$ are the higher order curvature terms given by

$$
\begin{aligned}
\rho^{(\mathrm{hc})} & =\frac{\rho^{(\mathcal{G T})}}{8 \pi \mathbb{G}}=\frac{1}{8 \pi G \mathfrak{B}}\left[\frac{1}{2}\left(f_{1}(\mathcal{G})+f_{2}(\mathcal{G}) f_{3}(T)\right)-\frac{1}{2} \mathcal{G} \Delta+12 H\left(H^{2}+\frac{\mathcal{K}}{a^{2}}\right)\left(\Delta_{\mathcal{G}} \dot{\mathcal{G}}+\Delta_{T} \dot{T}\right)\right], \\
p^{(\mathrm{hc})} & =\frac{p^{(\mathcal{G T})}}{8 \pi \mathbb{G}}=\frac{1}{8 \pi G \mathfrak{B}}\left[-\frac{1}{2}\left(f_{1}(\mathcal{G})+f_{2}(\mathcal{G}) f_{3}(T)\right)+\frac{1}{2} \mathcal{G} \Delta-8 H\left(H^{2}+\dot{H}\right)\left(\Delta_{\mathcal{G}} \dot{\mathcal{G}}+\Delta_{T} \dot{T}\right)\right. \\
& \left.-4\left(H^{2}+\frac{\mathcal{K}}{a^{2}}\right)\left(\Delta_{\mathcal{G}} \ddot{\mathcal{G}}+\Delta_{T} \ddot{T}+2 \Delta_{\mathcal{G} T} \dot{\mathcal{G}} \dot{T}+\Delta_{\mathcal{G} G} \dot{\mathcal{G}}^{2}+\Delta_{T T} \dot{T}^{2}\right)\right],
\end{aligned}
$$

where $\Delta=f_{1}^{\prime}(\mathcal{G})+f_{2}^{\prime}(\mathcal{G}) f_{3}(T), \mathcal{G}=24\left(H^{2}+\dot{H}\right)\left(H^{2}+\frac{\mathcal{K}}{a^{2}}\right)$ and $H=\frac{\dot{a}}{a}$ is a Hubble parameter. The subscripts $\mathcal{G}$ and $T$ denote derivatives of $\Delta$ with respect to $\mathcal{G}$ and $T$, respectively whereas dot represents time derivative. 


\section{Laws of Thermodynamics}

In this section, we discuss the first as well as GSLT at the apparent horizon of homogeneous and isotropic universe in the background of $f(\mathcal{G}, T)$ gravity.

\subsection{First Law}

Here, we construct the FLT which is based on the concept of energy conservation for model (2). The relation

$$
h^{\alpha \beta} \partial_{\alpha} \chi \partial_{\beta} \chi=0,
$$

where $h_{\alpha \beta}=\operatorname{diag}\left(1, \frac{-a^{2}(t)}{1-\mathcal{K} r^{2}}\right)$ is a two-dimensional line element, provides the radius of apparent horizon for the FRW universe model as

$$
\chi_{\text {(ah) }}=\left(H^{2}+\frac{\mathcal{K}}{a^{2}}\right)^{-\frac{1}{2}} .
$$

To measure the infinitesimal change in apparent horizon radius, we take the derivative of the above equation with respect to time and using Eq.(6), it follows that

$$
d \chi_{\text {(ah) }}=4 \pi G\left(\rho^{(\mathrm{tot})}+p^{(\mathrm{tot})}\right) \chi_{\text {(ah) }}^{3} H \mathfrak{B} d t,
$$

where $d t$ represents the corresponding small time interval. The temperature on the apparent horizon is given by [1]

$$
\tau=\frac{\left|K_{s g}\right|}{2 \pi}
$$

where $K_{s g}=\frac{1}{2 \sqrt{-h}} \partial_{\alpha}\left(\sqrt{-h} h^{\alpha \beta} \chi_{\beta}\right)$ ( $h$ is the determinant of $\left.h_{\alpha \beta}\right)$ is the surface gravity. For homogeneous and isotropic universe model, we have

$$
K_{s g}=\frac{1}{\chi_{\text {(ah) }}}\left(1-\frac{\dot{\chi}_{\text {(ah) }}}{2 \chi_{\text {(ah) }} H}\right)=\frac{1}{2} \chi_{\text {(ah) }}\left(H^{2}+\dot{H}+\frac{\mathcal{K}}{a^{2}}\right) .
$$

Bekenstein-Hawking entropy is measured in units of Newton's gravitational constant defined as one fourth of area of apparent horizon $\left(A=4 \pi \chi_{\text {(anh) }}^{2}\right)$ [8]. The entropy of stationary black hole solutions with bifurcate Killing horizons in the context of modified gravitational theories is a Noether charge entropy also dubbed as Wald entropy [9]. Brustein et al. [10] presented that this entropy is equal to quarter of apparent horizon area in units of effective gravitational coupling in these modified theories. Wald entropy in $f(G, T)$ gravity is given by

$$
S=\frac{A}{4 G^{\mathfrak{B}}}\left(1-\frac{4 \Delta}{\chi_{\text {(ah) }}^{2}}\right) .
$$

It is worth mentioning here that the entropy in $f(\mathcal{G})$ gravity is obtained for $f_{3}(T)=0$ while this formula corresponds to GR for $\Delta=0$ with $\mathfrak{B}=1$ [11]. Using Eqs.(8)-(10), it follows that

$$
\begin{aligned}
\tau d S & =4 \pi \chi_{\text {(ah) }}^{3}\left(\rho^{(\mathrm{tot})}+p^{(\mathrm{tot})}\right) H d t-2 \pi \chi_{\text {(ah) }}^{2}\left(\rho^{(\mathrm{tot})}+p^{(\mathrm{tot})}\right) d \chi_{\text {(ah) }}-\frac{4 \pi \tau}{G \mathfrak{B}} d \Delta \\
& +\frac{\pi \chi_{\text {(ah) }}^{2} \tau}{G}\left(1-\frac{4 \Delta}{\chi_{\text {(ah) }}^{2}}\right) d\left(\frac{1}{\mathfrak{B}}\right) .
\end{aligned}
$$


The total energy inside $\chi_{\text {(ah) }}$ for homogeneous and isotropic universe model is

$$
\mathcal{E}=V \rho^{(\mathrm{tot})}=\frac{4}{3} \pi \chi_{\text {(ah) }}^{3} \rho^{(\mathrm{tot})}=\frac{3 V}{8 \pi \mathbb{G}}\left(H^{2}+\frac{\mathcal{K}}{a^{2}}\right) .
$$

Using Eqs.(11) and (12), we obtain

$$
\tau d S=-d \mathcal{E}+W d V-\frac{4 \pi \tau}{\mathfrak{B} G} d \Delta+\frac{\chi_{\text {(ah) }}}{2 G}\left[1+2 \pi \chi_{\text {(ah) }} \tau\left(1-\frac{4 \Delta}{\chi_{\text {(ah) }}^{2}}\right)\right] d\left(\frac{1}{\mathfrak{B}}\right),
$$

where the infinitesimal change $d \mathcal{E}$ is caused by the small displacement in horizon radius $\chi_{\text {(ah) }}$ and $W=\frac{\rho^{(\mathrm{tot})}-p^{(\mathrm{tot})}}{2}$ measures the work done by the system. The FLT for model (2) can be expressed as

$$
\tau\left(d S+d_{i} S\right)=-d \mathcal{E}+W d V
$$

where

$$
d_{i} S=\frac{4 \pi}{\mathfrak{B} G} d \Delta-\frac{\chi_{(\mathrm{ah})}}{2 G \tau}\left[1+2 \pi \chi_{\text {(ah) }} \tau\left(1-\frac{4 \Delta}{\chi_{\text {(ah) }}^{2}}\right)\right] d\left(\frac{1}{\mathfrak{B}}\right),
$$

is the entropy production term which demonstrates the non-equilibrium behavior of thermodynamics. This shows that the field equations for model (2) do not meet with the universal form of FLT ( $d \mathcal{E}=$ $\tau d S+W d V)$ due to the presence of this auxiliary term.

\subsection{Generalized Second Law}

The GSLT is associated with the total entropy of the system and is defined as

$$
\dot{S}+\dot{S}_{(\text {tot })}+d_{i} \dot{S} \geq 0,
$$

where $d_{i} \dot{S}=\partial_{t}\left(d_{i} S\right)$ and $S_{\text {(tot) }}$ is associated with the entropy due to all matter contents present inside the horizon. The relationship between total entropy, energy density and pressure in a form of differential equation (Gibbs equation) is given by [4]

$$
\tau_{(\mathrm{tot})} d S_{(\mathrm{tot})}=d\left(\rho^{(\mathrm{tot})} V\right)+p^{(\mathrm{tot})} d V
$$

where $\tau_{\text {(tot) }}$ measures the total temperature of all contents within the horizon and is related to the temperature at the apparent horizon as

$$
\tau_{\text {(tot) }}=\lambda \tau, \quad 0<\lambda<1
$$

This shows that the apparent horizon temperature is always greater than the total temperature inside the horizon. Susbtituting Eqs.(14) and (16) in (15), we have

$$
\dot{S}+\dot{S}_{(\text {tot })}+d_{i} \dot{S}=\left(\frac{24+\chi_{\text {(ah) }}^{4} \mathcal{G}}{96 \pi \lambda \chi_{\text {(ah) }}}\right)\left[\left(\rho^{(\mathrm{tot})}+p^{(\mathrm{tot})}\right)\left(1-\frac{\lambda}{2}\right) \dot{V}+(1-\lambda) V \dot{\rho}^{(\mathrm{tot})}\right] \geq 0,
$$

Using Eqs.(5) and (6), this inequality becomes

$$
\left(\frac{24+\chi_{\text {(ah) }}^{4} \mathcal{G}}{192 \pi \lambda \mathfrak{B} G}\right) \Omega \chi_{\text {(ah) }}^{4} \geq 0,
$$


where

$$
\Omega=(2-\lambda) H\left(\dot{H}-\frac{\mathcal{K}}{a^{2}}\right)^{2}+\frac{2 H(1-\lambda)}{\chi_{\text {(ah) }}^{2}}\left(\dot{H}-\frac{\mathcal{K}}{a^{2}}\right)+\frac{(1-\lambda) \mathfrak{B}}{\chi_{\text {(ah) }}^{4}} \partial_{t}\left(\frac{1}{\mathfrak{B}}\right) .
$$

The GSLT is protected for $\mathcal{G}>0, \mathfrak{B}>0$ and $\Omega>0$. For flat FRW universe model, the inequalities $\mathcal{G}>0, H>0, \dot{H}>0, \mathfrak{B}>0$ and $\partial_{t}\left(\frac{1}{\mathfrak{B}}\right)>0$ must be fulfilled for the validity of GSLT. The equilibrium description of thermodynamics is evaluated at $\lambda=1$ as

$$
\chi_{\text {(ah) }}^{4} H\left(\dot{H}-\frac{\mathcal{K}}{a^{2}}\right)^{2}\left(\frac{24+\chi_{(\text {ah) }}^{4} \mathcal{G}}{192 \pi \lambda \mathcal{B} G}\right) \geq 0,
$$

which holds for positive values of $\mathcal{G}, H$ and $\mathfrak{B}$.

\section{Concluding Remarks}

In this paper, we have explored the first and GSLT in the background of $f(\mathcal{G}, T)$ gravity. These laws are discussed for the non-equilibrium thermodynamical picture at the apparent horizon of isotropic and homogeneous universe model. It is observed that total entropy is a combination of horizon entropy as well as an auxiliary entropy production term in FLT. The non-equilibrium thermodynamical behavior interprets energy exchange between inside and outside the apparent horizon leading to the additional entropy term. To check the validity of GSLT, we have constructed the general expression for model (2) in terms of horizon entropy, total entropy due to all matter contents inside the horizon and entropy production term. It is concluded that viability condition for this law is found to be achieved relative to universal condition in the context of modified gravitational theories [4].

\section{References}

[1] Cai, R.G. and Kim, S.P., J. High Energy Phys. 02, 050 (2005).

[2] Akbar, M. and Cai, R.G., Phys. Rev. D 75, 084003 (2007).

[3] Eling, C., Guedens, R. and Jacobson, T., Phys. Rev. Lett. 96, 121301 (2006); Cai, R.G. and Cao, L.M., Phys. Rev. D 75, 064008 (2007); Akbar, M. and Cai, R.G., Phys. Lett. B 648, 243 (2007); Sheykhi, A., Wang, B. and Cai, R.G., Nucl. Phys. B 779, 1 (2007); Cai, R.G. et al., Phys. Rev. D 78, 124012 (2008).

[4] Wu, S.F. et al., Class. Quantum Grav. 25, 235018 (2008).

[5] Sadjadi, H.M., Europhys. Lett. 92, 50014 (2010).

[6] Abdolmaleki, A. and Najafi, T., Int. J. Mod. Phys. D 25, 1650040 (2016).

[7] Sharif, M. and Ikram, A., Eur. Phys. J. C 76, 640 (2016).

[8] Bardeen, J.M., Carter, B. and Hawking, S.W., Commun. Math. Phys. 31, 161 (1973); Bekenstein, J.D., Phys. Rev. D 7, 2333 (1973); Hawking, S.W., Commun. Math. Phys. 43, 199 (1975); ibid. 46, 206 (1976).

[9] Wald, R.M., Phys. Rev. D 48, R3427 (1993).

[10] Brustein, R., Gorbonos, D. and Hadad, M., Phys. Rev. D 79, 0444025 (2009).

[11] Sadjadi, H.M., Phys. Scr. 83, 055006 (2011); Sharif, M. and Fatima, I., Astrophys. Space Sci. 354, 507 (2014). 